Research Article

\title{
Iterative Analysis of Nonlinear BBM Equations under Nonsingular Fractional Order Derivative
}

\author{
Gauhar Ali, ${ }^{1}$ Israr Ahmad, ${ }^{1}$ Kamal Shah $\mathbb{D D}^{1},{ }^{1}$ and Thabet Abdeljawad $\mathbb{D}^{2,3,4}$ \\ ${ }^{1}$ Department of Mathematics, University of Malakand, Chakdara Dir (Lower), Khyber Pakhtunkhwa, Pakistan \\ ${ }^{2}$ Department of Mathematics and General Sciences, Prince Sultan University, Riyadh, Saudi Arabia \\ ${ }^{3}$ Department of Medical Research, China Medical University, Taichung 40402, Taiwan \\ ${ }^{4}$ Department of Computer Science and Information Engineering, Asia University, Taichung, Taiwan ”
}

Correspondence should be addressed to Thabet Abdeljawad; tabdeljawad@psu.edu.sa

Received 13 May 2020; Revised 16 June 2020; Accepted 23 June 2020; Published 13 August 2020

Academic Editor: Zine El Abiddine Fellah

Copyright (c) 2020 Gauhar Ali et al. This is an open access article distributed under the Creative Commons Attribution License, which permits unrestricted use, distribution, and reproduction in any medium, provided the original work is properly cited.

\begin{abstract}
The present research work is devoted to investigate fractional order Benjamin-Bona-Mahony (FBBM) as well as modified fractional order FBBM (FMBBM) equations under nonlocal and nonsingular derivative of Caputo-Fabrizio (CF). In this regards, some qualitative results including the existence of at least one solution are established via using some fixed point results of Krasnoselskii and Banach. Further on using an iterative method, some semianalytical results are also studied. The concerned tool is formed when the Adomian decomposition method is coupled with some integral transform like Laplace. Graphical presentations are given for various fractional orders. Also, the concerned method is also compared with some variational-type perturbation method to demonstrate the efficiency of the proposed method.
\end{abstract}

\section{Introduction}

Fractional calculus is the generalized form of classical calculus. With the rapid change in science and technology, the aforesaid area has attracted the attention of many researchers. The mentioned branch has many applications in different areas of science like modeling, control theory, physics, signal processing, economics, and chemistry [1-4]. Different researchers have studied fractional differential equations (FODEs) in their own way, including the stability aspect, qualitative theory, optimization, and numerical simulations. Many real-world problems are nonlinear in nature, and their investigation is important for fruitful information. Therefore, researchers have studied various problems of FODEs by using different techniques and methods. One of the important aspects is the existence theory of solution which has given proper attention in the last years [5-11]. By using the fixed point theory, the existence theory to numerous problems has been established [12-16]. The authors in [17-22] also studied different aspects of FODEs using a derivative with nonsingular kernel and Laplace transform. Therefore, we intend to establish the aforementioned theory for the following problem with $[0, \tau]=J$

$$
\begin{aligned}
{ }^{C F} D_{t}^{\gamma} v(t, y)-h(t, v(t, y)) & =0, \\
v(y, 0) & =f(y),
\end{aligned}
$$

where $h: J \times R \longrightarrow R$ and $f \in C(J)$. The existence of at least one solution of (1) has been studied with the help of a fixed point approach, since the differential operator involving fractional order have a great degree of freedom. Therefore, it comprehensively describes many dynamical properties and characteristic of various processes/phenomena [23, 24]. Then, we establish an algorithm to compute the approximate analytical solutions for the following cases of BBM equations with $y, t \in J, \gamma \in(0,1]$ as 
Case 1.

$$
\begin{aligned}
{ }^{C F} D_{t}^{\gamma} v(t, y)-v_{y y t}(t, y)+a v(t, y) v_{y}(t, y) & =0, \\
v(0, y) & =f(y) .
\end{aligned}
$$

Case 2.

$$
\begin{aligned}
{ }^{C F} D_{t}^{\gamma} v(t, y)+v_{y}(t, y)+a v(t, y) v_{y}(t, y)+v_{y y y}(t, y) & =0 \\
v(y, 0) & =f(y) .
\end{aligned}
$$

Case 3.

$$
\begin{aligned}
{ }^{C F} D_{t}^{\gamma} v(t, y)+v_{y}(t, y)+a v^{2}(t, y) v_{y}(t, y)+v_{y y y}(t, y) & =0, \\
v(y, 0) & =f(y) .
\end{aligned}
$$

where $a$ is a real constant. The abovementioned problems are also called regularized long-wave equation which is the improved form of the Korteweg-de Vries equation (KDVE). Such equation has been largely used for modeling of waves of small amplitudes and in the soliton theory of fractals and dynamics. Moreover, KDVE has countless integrals of motion and BBM has only three [25-32]. For generalized $n$ -dimensional BBM equation and its applications, we refer to $[25,33,34]$. The aforementioned equation has been studied in surface waves of a long period of fluid [26]. Also, for the dynamic aspect of the BBM equation, we refer [35]. The mentioned equation is not only suitable for superficial waves but also for acoustic and hydromagnetic waves; because of this, the BBM equation has upper hand on KDVE. We enrich our study by investigating the modified form of BBM equation abbreviated as MBBM [36]. We use the decomposition method coupled with Laplace transform to establish series solution to our proposed problems (2), (3), and (4). The mentioned problems have been studied by the homotopy perturbation method (HPM), variational method (VHPM), wavelet method, etc., but these studies are limited to fractional order derivative involving the usual Caputo and integer order derivative. To the best of authors' information, no study exists in the present literature to address the investigation of the aforesaid problems under nonsingular CF derivative. The mentioned derivative was introduced in 2016 and has been found suitable in applications of many thermal problems. The concerned nonlocal integral of CF for a function is the average of the function and its Riemann integral which works as a filter, for various applications of the concerned derivative, we refer to $[12,13,18,19]$. So far, we know that there is no investigation present in the literature which addresses the study of the mentioned problems under nonlocal and nonsingular kernel derivatives with fractional order. We establish some qualitative results of the existence of at least one solution by Krasnoselskii and Banach fixed point results. Further, by the proposed method of Laplace transform coupled with Adomian decomposition (LADM), we compute the series solution whose convergence is also stud- ied. Also, the results are compared with the results of VHPM. The results reveal that the proposed method can also be used as a powerful tool to find approximate results to many nonlinear problems.

\section{Preliminaries}

Definition 1 (see [37]). Let $v \in H^{1}(0, a), a>0, \gamma \in(0,1)$, then $\mathrm{CF}$ derivative is defined below

$$
\begin{array}{r}
{ }^{C F} D_{t}^{\gamma} v(t)=\frac{\mathbb{N}(\gamma)}{(1-\gamma)} \int_{0}^{t} \exp \left[-\frac{\gamma(t-s)}{1-\gamma}\right] v^{\prime}(\mu) d \eta \\
\gamma \in(0,1), t \geq 0
\end{array}
$$

where the function $\mathbb{N}(\gamma)$ is called normalization.

Definition 2 (see [38]). The CF integral with $\gamma \in(0,1)$ is given below

$$
{ }^{C F} \mathbf{J}_{t}^{\gamma} v(t)=\frac{(1-\gamma) v(t)}{\mathbb{N}(\gamma)}+\frac{\gamma}{\mathbb{N}(\gamma)} \int_{0}^{t} v(\eta) d \eta
$$

Definition 3 (see [37]). For the CF derivative of order $\gamma \in(0,1]$ and $n \in \mathbb{N}$, the Laplace transform is given below

$$
\begin{aligned}
\mathscr{L} & \left({ }^{C F} D_{t}^{n+\gamma} v(t)\right)(s) \\
= & \frac{1}{(1-\gamma)} \mathscr{L}\left(v^{(n+1)}(t)\right) \mathscr{L}\left(\exp \left(-\frac{\gamma t}{1-\gamma}\right)\right) \\
= & \frac{s^{n+1} \mathscr{L}[v(t)]-s^{n} v(0)-s^{n-1} v^{\prime}(0)-\cdots-v^{n}(0)}{s+\gamma(1-s)} .
\end{aligned}
$$

Definition 4. The considered method is used to compute the solution in an infinite series form. We consider the solution as

$$
v(t, y)=\sum_{n=0}^{\infty} v_{n}(y, t)
$$

and nonlinear term is decompose as

$$
\mathscr{N} v=\sum_{n=0}^{\infty} \mathscr{A}_{n}
$$

where $\mathscr{A}_{n}$ is given by

$$
\mathscr{A}_{n}=\frac{1}{\Gamma(n+1)} D_{\mu}^{n}\left[\mathcal{N}\left(\sum_{j=0}^{n} \mu^{j} v_{j}\right)\right]_{\mu=0} .
$$

Theorem 5 (Krasnoselskii's fixed point theorem [39]). If $D \subset X$ be a convex and closed nonempty subset, there exist two operators $\mathscr{G}_{1}$ and $\mathscr{G}_{2}$ such that

(i) $\mathscr{G}_{1} v_{1}+\mathscr{G}_{2} v_{2} \in D$ for all $v_{1}, v_{2} \in D$

(ii) $\mathscr{G}_{1}$ is a condensing operator 
(iii) $\mathscr{G}_{2}$ is continuous and compact

then, there exists at least one solution $v \in D$ which satisfies $\mathscr{G}_{1}(v)+\mathscr{G}_{2}(v)=v$.

\section{Steps for Existence of Results}

In the ongoing section, we discuss the existence of the considered problem.

Lemma 6. Under Definitions (1) and (2), we have

$$
\begin{aligned}
v(t, y)= & f(y)+\frac{(1-\gamma)}{\mathbb{N}(\gamma)}[h(t, v(t, y)) \\
& -h(0, v(0, y))] \frac{\gamma}{\mathbb{N}(\gamma)} \int_{0}^{t} h(\theta, v(\theta, y)) d \theta
\end{aligned}
$$

The assumptions needed for our work are

$\left(B_{1}\right) h(t, v)$ is the nonlinear function satisfy the growth condition as

$$
|h(t, v)| \leq b_{h}+C|v|^{p}, p \in(0,1), C \geq 0 .
$$

$\left(B_{2}\right)$ For all $v_{1}, v_{2} \in$ Rthere exist a positive constant $k_{h}$ one can get,

$$
\left|h\left(t, v_{1}\right)-h\left(t, v_{2}\right)\right| \leq k_{h}\left|v_{1}-v_{2}\right|, \quad \text { for all } t \in \mathscr{J} .
$$

Furthermore, $h(t, 0)=0$ holds.

$\mathscr{G}_{1}, \mathscr{G}_{2}: X \longrightarrow X$ are the operators defined as

$$
\left\{\begin{array}{l}
\mathscr{G}_{1}(t, v)=f(y)+\frac{(1-\gamma)}{\mathbb{N}(\gamma)}[h(t, v(t, y))-h(0, v(0, y))], \\
\mathscr{G}_{2}(t, v)=\frac{\gamma}{\mathbb{N}(\gamma)} \int_{0}^{t} h(\theta, v(\theta, y)) d \theta .
\end{array}\right.
$$

Theorem 7. In light of hypothesis $\left(B_{1}\right)$ and $\left(B_{2}\right)$, if $(1-\gamma)$ / $(\mathbb{N}(\gamma)) k_{h} \leq 1$, then (1) has at least one solution.

Proof. Using (2.5), and a bounded set defined as $D=\{v \in$ $\left.X:\|v\|_{X} \leq R\right\}$. The continuity of $v(t, y)$ implies that $\mathscr{G}_{1}$ and $\mathscr{G}_{2}$ are continuous operators. To show that $\mathscr{G}_{1}$ is a condensing map, $\operatorname{consider} v_{1}, v_{2} \in D$, under the assumption $\left(B_{1}\right)$

$$
\begin{aligned}
& \left\|\mathscr{G}_{1}\left(v_{1}\right)-\mathscr{G}_{1}\left(v_{2}\right)\right\|_{X} \\
& \quad=\max _{t \in \mathcal{J}}\left|\frac{(1-\gamma)}{\mathbb{N}(\gamma)} h\left(t, v_{1}(t, y)\right)-\frac{(1-\gamma)}{\mathbb{N}(\gamma)} h\left(t, v_{2}(t, y)\right)\right| \\
& \quad \leq \frac{(1-\gamma)}{\mathbb{N}(\gamma)} k_{h}\left\|v_{2}-v_{1}\right\|_{X} .
\end{aligned}
$$

This show that $G_{1}$ is a condensing map; further, for the continuity and compactness of $G_{2}$ for all $v \in D$, consider

$$
\begin{aligned}
\left\|\mathscr{G}_{2}(v)\right\|_{X} & =\max _{t \in \mathcal{J}}\left|\frac{\gamma}{\mathbb{N}(\gamma)} \int_{0}^{t} h(\theta, v(\theta, y)) d \theta\right| \\
& \leq \frac{\gamma}{\mathbb{N}(\gamma)} \max _{t \in \mathcal{J}} \int_{0}^{t}|h(\theta, v(\theta, y))| d \theta \\
& \leq \frac{\gamma}{\mathbb{N}(\gamma)} \int_{0}^{t}\left[b_{h}+C|v|^{p}\right] d \theta \\
& \leq \frac{\gamma}{\mathbb{N}(\gamma)}\left[b_{h}+C R^{P}\right] \tau .
\end{aligned}
$$

Therefore, $G_{2}$ is bounded on $D$. For continuity considering $t_{1}-t_{2}>0$, one can infer that

$$
\begin{aligned}
& \left|\mathscr{G}_{2} v_{1}\left(t_{1}\right)-\mathscr{G}_{2} v_{2}\left(t_{2}\right)\right| \\
& \quad=\left|\frac{\gamma}{\mathbb{N}(\gamma)}\left[\int_{0}^{t_{1}} h(\theta, v(\theta, y) d \theta)-\int_{0}^{t_{2}} h(\theta, v(\theta, y)) d \theta\right]\right| \\
& \quad \leq \frac{\gamma}{\mathbb{N}(\gamma)}\left[b_{h}+C R^{p}\right]\left(t_{1}-t_{2}\right) .
\end{aligned}
$$

This implies that $\left\|\mathscr{G}_{2} v_{1}\left(t_{1}\right)-\mathscr{G}_{2} v_{2}\left(t_{2}\right)\right\|_{X} \longrightarrow 0$, as $t_{1}$ tends to $t_{2}$. So it shows that $\mathscr{G}_{2}$ is compact and equicontinuous; by Theorem 1, the problem (1) has no less than one solution in $D$.

Theorem 8. In view of assumption $\left(B_{2}\right)$ if $((1+\gamma(\tau-1))$ / $(\mathbb{N}(\gamma))) k_{h}$, then problem (1) has a unique solution.

Proof. By using (1), we define the operator $G$ as

$$
\begin{aligned}
G v(t, y)= & f(y)+\frac{(1-\gamma)}{\mathbb{N}(\gamma)}[h(t, v(t, y))-h(0, v(0, y))] \\
& +\frac{\gamma}{\mathbb{N}(\gamma)} \int_{0}^{t} h(\theta, v(\theta, y)) d \theta
\end{aligned}
$$

Suppose $v_{1}, v_{2} \in X$, we have

$$
\begin{aligned}
\left\|G v_{1}-G v_{2}\right\| \backslash \text { leq } x \\
\leq \max _{t \in \mathcal{J}}\left|\frac{(1-\gamma)}{\mathbb{N}(\gamma)}\left[h\left(t, v_{1}(t, y)-h\left(t, v_{2}(t, y)\right)\right)\right]\right| \\
\quad+\max _{t \in \mathcal{J}} \int_{0}^{t}\left[h\left(\theta, v_{1}(\theta, y)\right)-h\left(\theta, v_{2}(\theta, y)\right) d \theta\right] \\
\leq \frac{(1-\gamma)}{\mathbb{N}(\gamma)} k_{h}\left\|v_{1}-v_{2}\right\|_{X}+\frac{\gamma}{\mathbb{N}(\gamma)} \tau k_{h}\left\|v_{1}-v_{2}\right\|_{X} \\
=\left(\frac{1+\gamma(\tau-1)}{\mathbb{N}(\gamma)}\right) k_{h}\left\|v_{1}-v_{2}\right\|_{X} .
\end{aligned}
$$

Therefore, $G$ is a condensing operator which implies the uniqueness of solution. 


\section{Main Results}

To present the iterative solution of our considered problem, we first give a general procedure for the given problem as

$$
\left\{\begin{array}{l}
{ }^{C F} D_{t}^{\gamma} v(t, y)=\mathscr{N} v(t, y)+\mathscr{R} v+g(t, y) \\
v(0, y)=f(y) y \in[0,1]
\end{array}\right.
$$

where $\mathcal{N}$ is a nonlinear operator and $\mathscr{R}$ is a linear operator and $g$ is external source function. Further, $f: J \longrightarrow R$ is a nonlocal, bounded, and continuous function.

Taking Laplace transform of (14) and using the initial condition, we have

$$
\begin{aligned}
{ }^{C F} D_{t}^{\gamma}[v(t, y)]= & \frac{f(y)}{s}+\frac{s+\gamma(1-s)}{s} \mathscr{L}[N v(t, y) \\
& +\mathscr{R} v(t, y)+g(t, y)] .
\end{aligned}
$$

Let us consider the solution in terms of a series as

$$
v=\sum_{n=0}^{\infty} v_{n}
$$

and decompose the nonlinear term $\mathcal{N} v(t, y)$ in terms of the Adomian polynomial as

$$
\mathcal{N} v=\sum_{n=0}^{\infty} \mathscr{A}_{n}
$$

where

$$
\mathscr{A}_{n}=\left.\frac{1}{\Gamma(n+1)} D_{u}^{n}\left[\mathcal{N}\left(\sum_{j=0}^{n} \mu^{j} v_{j}\right)\right]\right|_{\mu=0}
$$

Using (15) and comparing the terms on both sides, we have

$$
\begin{aligned}
& v_{0}=f(y), \\
& v_{1}=\mathscr{L}^{-1}\left[\frac{\gamma+s(1-\gamma)}{s} \mathscr{L}\left(\mathscr{A}_{0}(t, y) \mathscr{R} v_{0}(t, y)+g(t, y)\right)\right], \\
& \vdots \\
& v_{n+1}=\mathscr{L}^{-1}\left[\frac{\gamma+s(1-\gamma)}{s} \mathscr{L}\left(\mathscr{A}_{n}(t, y) \mathscr{R} v_{n}(t, y)+g(t, y)\right)\right], \quad n \geq 0 .
\end{aligned}
$$

After evaluation, the required solution is

$$
v(t, y)=\sum_{n=0}^{\infty} v_{n}(t, y)=v_{0}(t, y)+v_{1}(t, y)+v_{2}(t, y)+\cdots
$$

Theorem 9. Let $T$ be a nonlinear contractive operator on a Banach space $X$, such that for all $v, v^{*} \in X$, one has

$$
\left\|T v-T v^{*}\right\|_{X} \leq k\left\|v-v^{*}\right\|_{X}, \quad 0<k<1 .
$$

Then, the unique fixed point $v$ satisfies the relation $T v=v$. Let us write the generated series (26) as

$$
v_{n}=T\left(v_{n-1}\right), v_{n-1}=\sum_{i=0}^{n-1} v_{i}, \quad n=1,2,3, \cdots,
$$

and assume that $v_{0} \in S_{r}(v)$, where $S_{r}(v)=\left\{v^{*} \in X:\left\|v-v^{*}\right\|_{X}\right.$ $\leq r, r \geq 0\}$. Then, we have

$$
\begin{aligned}
& \left(A_{1}\right) x_{n} \in S_{r}(v) . \\
& \left(A_{2}\right) \lim _{n \rightarrow \infty} v_{n}=v .
\end{aligned}
$$

Proof. $\left(A_{1}\right)$ By using mathematical induction for $n=1$, we have

$$
\left\|v_{1}-v\right\|_{X}=\left\|T v_{0}-T v\right\|_{X} \leq\left\|\mathrm{k} v_{0}-v\right\|_{X} .
$$

Considering that the result for $n-1$ is true, then

$$
\left\|v_{n-1}-v\right\|_{X} \leq k^{n-1}\left\|v_{0}-v\right\|_{X}
$$

Now consider

$$
\left\|v_{n}-v\right\|_{X}=\left\|T v_{n-1}-T v\right\|_{X} \leq k\left\|v_{n-1}-v\right\|_{X} \leq k^{n}\left\|v_{0}-v\right\|_{X} .
$$

With the help of $\left(A_{1}\right)$, we have

$$
\left\|v_{n}-v\right\|_{X} \leq k^{n}\left\|v_{0}-v\right\|_{X} \leq k^{n} r \leq r
$$

which gives that $v_{n} \in S_{r}(v)$, since

$$
\left\|v_{n}-v\right\|_{X} \leq k^{n}\left\|v_{0}-v\right\|_{X}
$$

and $\lim _{n \rightarrow \infty} k^{n}=0$. Therefore, we have $\lim _{n \rightarrow \infty}\left\|v_{n}-v\right\|_{X}=0$ which yields $\lim _{n \rightarrow \infty} v_{n}=v$.

4.1. General Procedure for Case 1. Consider the following FBBM equation under the given condition as

$$
\left\{\begin{array}{l}
{ }^{C F} D_{t}^{\gamma} v(t, y)-v_{y y t}(t, y)+a v(t, y) v_{y}(t, y)=0, \\
v(0, y)=f(y) .
\end{array}\right.
$$

Taking Laplace transform of (35), one has

$\mathscr{L}[v(t, y)]=\frac{f(y)}{s}+\frac{\gamma+s(1-\gamma)}{s} \mathscr{L}\left[v_{y y t}(t, y)-a v(t, y) v_{y}(t, y)\right]$

Let us consider the solution in terms of a series as

$$
v=\sum_{n=0}^{\infty} v_{n}
$$


and the decomposition of the nonlinear term is

$$
v v_{y}=\sum_{n=0}^{\infty} \mathscr{A}_{n}
$$

where

$$
\mathscr{A}_{n}=\left.\frac{1}{\Gamma(n+1)} D_{\mu}^{n}\left[\left(\sum_{j=0}^{n} \mu^{j} v_{j}\right)\left(\sum_{j=0}^{n} \mu^{j} v_{j y}\right)\right]\right|_{\mu=0} .
$$

$\mathscr{A}_{n}$ for different values of $n$ are

$$
\begin{aligned}
& \mathscr{A}_{0}=v_{0}(t, y) v_{0 y}(t, y), \\
& \mathscr{A}_{1}=v_{0}(t, y) v_{1 y}(t, y)+v_{0 y}(t, y) v_{1}(t, y),
\end{aligned}
$$

and so on. Putting these values in (36) and comparing the terms on both sides, we have

$$
\begin{aligned}
& v_{0}(t, y)=f(y), \\
& v_{1}(t, y)=\mathscr{L}^{-1}\left[\frac{\gamma+s(1-\gamma)}{s} \mathscr{L}\left(v_{0 y y t}(t, y)-a v_{0}(t, y) v_{0 y}(t, y)\right)\right], \\
& \vdots \\
& v_{n+1}(t, y)=\mathscr{L}^{-1}\left[\frac{\gamma+s(1-\gamma)}{s} \mathscr{L}\left(v_{n y y t}(t, y)-a v_{n}(t, y) v_{n y}(t, y)\right)\right], \quad n \geq 0 .
\end{aligned}
$$

After calculation, the solution of the considered problem (35) is obtained in the form of a series.

4.2. General Procedure for Case 2. Consider the following FBBM equation under the given condition as

$$
\left\{\begin{array}{l}
{ }^{C F} D_{t}^{v} v(t, y)+v_{y}(t, y)+a v(t, y) v_{y}(t, y)+v_{y y y}(t, y)=0 \\
v(0, y)=f(y)
\end{array}\right.
$$

Taking Laplace of (42), one may have

$$
\begin{aligned}
\mathscr{L}[v(t, y)]= & \frac{f(y)}{s}-\frac{\gamma+s(1-\gamma)}{s} \mathscr{L}[v(t, y) \\
& \left.+v_{y y y}(t, y)+a v(t, y) v_{y}(t, y)\right] .
\end{aligned}
$$

Here, we consider the unknown solution as

$$
v=\sum_{n=0}^{\infty} v_{n}
$$

and the nonlinear term is decomposed as

$$
v(t, y) v_{y}=\sum_{n=0}^{\infty} \mathscr{A}_{n}
$$

where $\mathscr{A}_{n}$ is define as

$$
\mathscr{A}_{n}=\left.\frac{1}{\Gamma(n+1)} D_{\mu}^{n}\left[\left(\sum_{j=0}^{n} \mu^{j} v_{j}\right)\left(\sum_{j=0}^{n} \mu^{j} v_{j y}\right)\right]\right|_{\mu=0} .
$$

$\mathscr{A}_{n}$ for different values of $n$ are

$$
\begin{aligned}
& \mathscr{A}_{0}=v_{0}(t, y) v_{0 y}(t, y), \\
& \mathscr{A}_{1}=v_{0}(t, y) v_{1 y}(t, y)+v_{0 y}(t, y) v_{1}(t, y),
\end{aligned}
$$

and so on. Using these values in equation (43) and equating the corresponding terms on both sides, we have

$$
\begin{aligned}
& v_{0}(t, y)=f(y), \\
& v_{1}=-\mathscr{L}^{-1}\left[\frac{\gamma+s(1-\gamma)}{s} \mathscr{L}\left(v_{0}(t, y)+v_{0 y y y}(t, y)+a \mathscr{A}_{0}\right)\right], \\
& v_{2}(t, y)=-\mathscr{L}^{-1}\left[\frac{\gamma+s(1-\gamma)}{s} \mathscr{L}\left(v_{1}(t, y)+v_{1 y y y}(t, y)+a \mathscr{A}_{1}\right)\right], \\
& \vdots \\
& v_{n+1}(t, y)=-\mathscr{L}^{-1}\left[\frac{\gamma+s(1-\gamma)}{s} \mathscr{L}\left(v_{n}(t, y)+v_{n y y y}(t, y)+a \mathscr{A}_{n}\right)\right], \quad n \geq 0 .
\end{aligned}
$$

In this way, the series solution of the proposed problem (42) is obtained.

4.3. Procedure for Case 3. Consider the following FMBBM equation under the given condition

$$
\left\{\begin{array}{l}
{ }^{C F} D_{t}^{\gamma} v(t, y)+v_{y}(t, y)+a v^{2}(t, y) v_{y}(t, y)+v_{y y y}(t, y)=0, \\
v(0, y)=f(y) .
\end{array}\right.
$$

Taking Laplace of (49) and after rearranging the terms, we have

$$
\begin{aligned}
\mathscr{L}[v(t, y)]= & \frac{f(y)}{s}-\frac{\gamma+s(1-\gamma)}{s} \mathscr{L}[v(t, y) \\
& \left.+v_{y y y}(t, y)+a v^{2}(t, y) v_{y}(t, y)\right] .
\end{aligned}
$$

Here, we consider the unknown $v(t, y)$ as

$$
v=\sum_{n=0}^{\infty} v_{n},
$$

and nonlinear term is decomposed as

$$
v^{2}(t, y) v_{y}=\sum_{n=0}^{\infty} \mathscr{A}_{n}
$$


where $\mathscr{A}_{n}$ is "Adomian polynomials" defined as

$$
\mathscr{A}_{n}=\frac{1}{\Gamma(n+1)} D_{\mu}^{n}\left[\left(\sum_{j=0}^{n} \mu^{j} v_{j}\right)^{2}\left(\sum_{j=0}^{n} \mu^{j} v_{j y}\right)\right]_{\mu=0} .
$$

$\mathscr{A}_{n}$ for different values of $n$ are

$$
\begin{aligned}
& \mathscr{A}_{0}=v_{0}^{2}(t, y) v_{0 y}(t, y), \\
& \mathscr{A}_{1}=v_{0}^{2}(t, y) v_{1 y}(t, y)+2 v_{0}(t, y) v_{0 y}(t, y) v_{1}(t, y),
\end{aligned}
$$

and so on. Putting these values in equation (50) and comparing terms on both sides, we have

$$
\begin{aligned}
& v_{0}(t, y)=g(y), \\
& v_{1}(t, y)=-\mathscr{L}^{-1}\left[\frac{\gamma+s(1-\gamma)}{s} L\left(v_{0}(t, y)+v_{0 y y y}(t, y)+a \mathscr{A}_{0}\right)\right], \\
& v_{2}(t, y)=-\mathscr{L}^{-1}\left[\frac{\gamma+s(1-\gamma)}{s} L\left(v_{1}(t, y)+v_{1 y y y}(t, y)+a \mathscr{A}_{1}\right)\right], \\
& \vdots \\
& v_{n+1}(t, y)=-\mathscr{L}^{-1}\left[\frac{\gamma+s(1-\gamma)}{s} L\left(v_{n}(t, y)+v_{n y y y}(t, y)+a \mathscr{A}_{n}\right)\right], \quad n \geq 0 .
\end{aligned}
$$

Hence, in this case, the solution in same way may be computed.

\section{Examples}

Here, in the ongoing section, we find series solutions for (35), (42), and (49) with the help of LADM using CFFOD.

Example 1. Consider the following FBBM equation [40] as

$$
\begin{aligned}
{ }^{C F} D_{t}^{\gamma} v(t, y) & =v_{y y t}(t, y)-v(t, y) v_{y}(t, y), \\
v(0, y) & =\sec h^{2}\left(\frac{y}{4}\right) .
\end{aligned}
$$

With the exact solution given below,

$$
v(t, y)=\sec h^{2}\left(\frac{y}{4}-\frac{t}{3}\right)
$$

With the help of the procedure discussed in Case 1, one has

$$
\begin{aligned}
v_{0}= & \sec h^{2}\left(\frac{y}{4}\right), \\
v_{1}= & -\frac{1}{2}(1+\gamma(t-1)) \sec h^{4}\left(\frac{y}{4}\right) \tanh \left(\frac{y}{4}\right), \\
v_{2}= & -\frac{\gamma}{2}(1+\gamma t-\gamma)\left[\sec h^{6}\left(\frac{y}{4}\right) \tanh ^{3}\left(\frac{y}{4}\right)\right. \\
& \left.+\frac{3}{2} \sec h^{8}\left(\frac{y}{4}\right) \tanh \left(\frac{y}{4}\right)\right] \\
& +\frac{1}{2}\left(1+\frac{(\gamma t)^{2}}{2}-2 \gamma^{2} t+\gamma^{2}\right) \\
& \cdot\left[\sec h^{6}\left(\frac{y}{4}\right) \tanh ^{2}\left(\frac{y}{4}\right)+\frac{1}{4} \sec h^{8}\left(\frac{y}{4}\right)\right. \\
& \left.+\frac{1}{2} \sec ^{6}\left(\frac{y}{4}\right) \tanh ^{2}\left(\frac{y}{4}\right)\right] .
\end{aligned}
$$

And hence, the solution of (56) in the form of a series is given by

$$
\begin{aligned}
v(t, y)= & \sec h^{2}\left(\frac{y}{4}\right)-\frac{1}{2}(1+\gamma(t, y)) \sec h^{4}\left(\frac{y}{4}\right) \tanh \left(\frac{y}{4}\right) \\
& -\frac{\gamma}{2}(1+\gamma t-\gamma)\left[\sec h^{6}\left(\frac{y}{4}\right) \tanh ^{3}\left(\frac{y}{4}\right)\right. \\
& \left.+\frac{3}{2} \sec h^{8}\left(\frac{y}{4}\right) \tanh \left(\frac{y}{4}\right)\right] \\
& +\frac{1}{2}\left(1+\frac{(\gamma t)^{2}}{2}-2 \gamma^{2} t+\gamma^{2}\right) \\
& \cdot\left[\sec h^{6}\left(\frac{y}{4}\right) \tanh ^{2}\left(\frac{y}{4}\right)+\frac{1}{4} \sec h^{8}\left(\frac{y}{4}\right)\right. \\
& \left.+\frac{1}{2} \sec ^{6}\left(\frac{y}{4}\right) \tanh ^{2}\left(\frac{y}{4}\right)\right] .
\end{aligned}
$$

The approximate solution graphs for various fractional orders are given in Figure 1. We see from graphs as the order $\gamma \longrightarrow 1$, the behavior of the surfaces of the solution tends to the integer order. If we put $\gamma=1$ in the approximate solution, we get the solution at the integer order. Now, we compare the four-term LADM solution with the four-term solution of VHPM given in [40] in Table 1 at $\gamma=1$. From Table 1, we see that the absolute error between exact solutions and four-term LADM solutions at the integer order is slightly good than the absolute error for the mentioned four-term solution by using the VHPM. As compared to VHPM, the LADM is simple and easy to use to handle various nonlinear partial differential equations.

Example 2. Consider the FBBM equation using CFFOD as

$$
\begin{aligned}
{ }^{C F} D_{t}^{\gamma} v(t, y)+v_{y}(t, y) & \\
+v(t, y) v_{y}(t, y)+v_{y y y}(t, y) & =0, \\
v(0, y) & =e^{y} .
\end{aligned}
$$




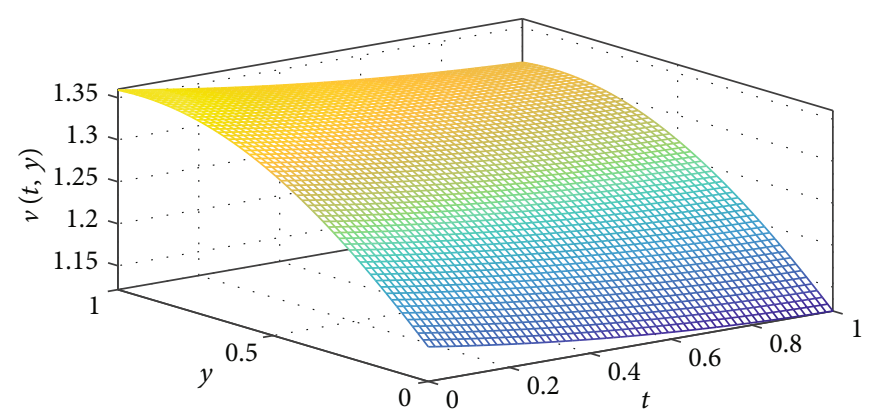

$(0.75)$

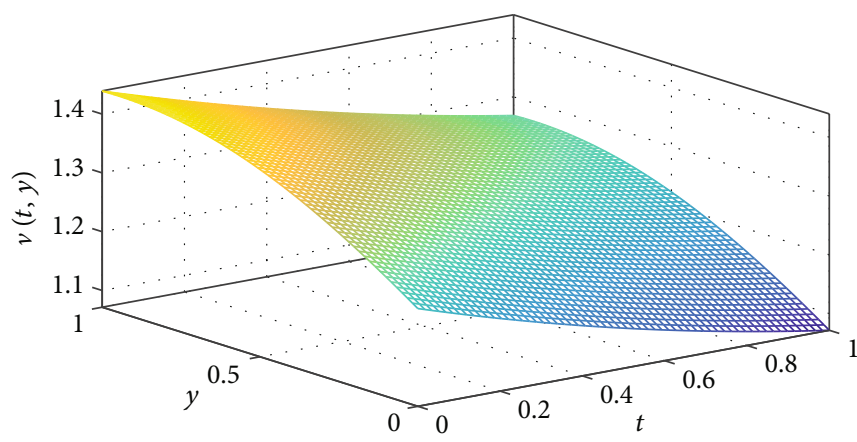

$\sqsupset(\gamma=0.95)$

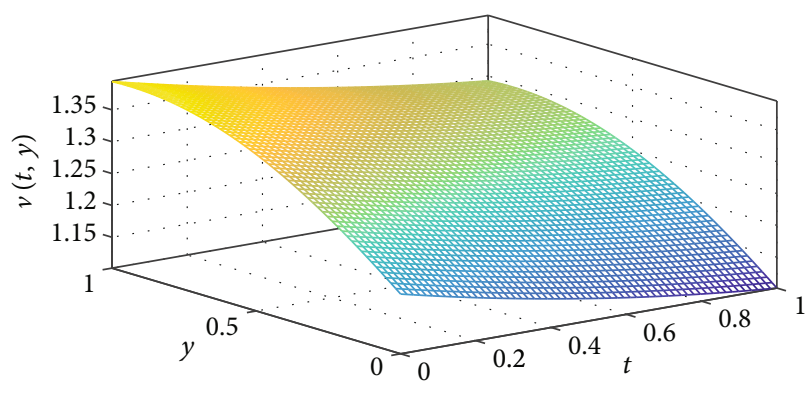

$(0.85)$

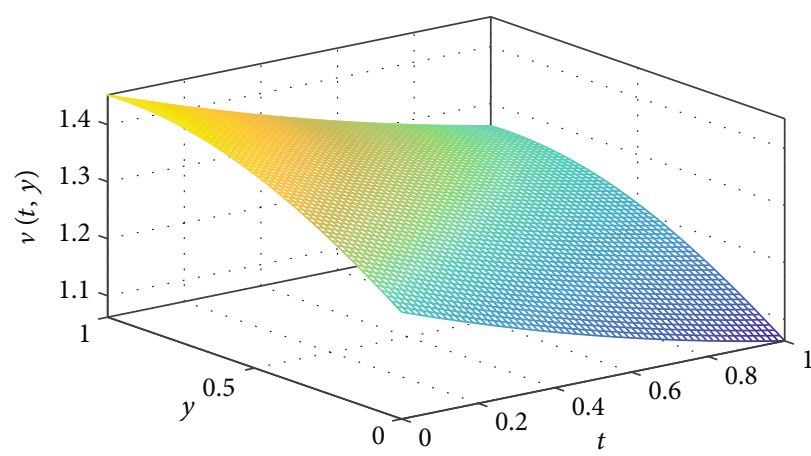

$(1.0)$

Figure 1: Surface plots of the required solution up to four terms at different values of $\gamma$ for Example 1.

TABle 1: Comparison between the absolute error at VHPM [40] and the present method (LADM) with the exact solution of Example 1.

\begin{tabular}{|c|c|c|c|c|c|c|}
\hline \multirow{2}{*}{$\begin{array}{l}y \longrightarrow \\
t\end{array}$} & \multicolumn{2}{|c|}{0.03} & \multicolumn{2}{|c|}{0.04} & \multicolumn{2}{|c|}{0.05} \\
\hline & VHPM & LADM & VHPM & LADM & VHPM & LADM \\
\hline 0.01 & $1.1543 \times 10^{-4}$ & $1.0534 \times 10^{-4}$ & $1.4926 \times 10^{-4}$ & $1.0067 \times 10^{-4}$ & $1.8307 \times 10^{-4}$ & $1.0546 \times 10^{-4}$ \\
\hline 0.02 & $2.5862 \times 10^{-4}$ & $1.0987 \times 10^{-4}$ & $3.2626 \times 10^{-4}$ & $2.4321 \times 10^{-4}$ & $3.9387 \times 10^{-4}$ & $2.0088 \times 10^{-4}$ \\
\hline 0.03 & $4.2956 \times 10^{-4}$ & $2.2345 \times 10^{-4}$ & $5.3101 \times 10^{-4}$ & $3.7054 \times 10^{-4}$ & $6.3239 \times 10^{-4}$ & $4.1234 \times 10^{-4}$ \\
\hline 0.04 & $6.2827 \times 10^{-4}$ & $3.1033 \times 10^{-4}$ & $7.6350 \times 10^{-4}$ & $3.4034 \times 10^{-4}$ & $8.9864 \times 10^{-4}$ & $6.6523 \times 10^{-4}$ \\
\hline 0.05 & $8.5474 \times 10^{-4}$ & $4.5643 \times 10^{-4}$ & $1.0237 \times 10^{-3}$ & $3.9876 \times 10^{-4}$ & $1.1926 \times 10^{-4}$ & $1.0195 \times 10^{-4}$ \\
\hline
\end{tabular}

With the help of procedure discussed for Case 2, one has

$$
\begin{aligned}
v_{0}= & e^{y}, \\
v_{1}= & -(1+\gamma t-\gamma)\left(2 e^{y}+e^{2 y}\right), \\
v_{2}= & {\left[(1-\gamma)^{2}+2 \gamma t(1-\gamma)+\frac{\gamma^{2} t^{2}}{2}\right]\left(4 e^{y}+14 e^{2 y}+3 e^{3 y}\right), } \\
v_{3}= & -\left[(1-\gamma)^{3}+3 \gamma t\left(1-\gamma^{2}\right)+\frac{3}{2} \gamma^{2}(1-\gamma) t^{2}+\frac{\gamma^{3} t^{3}}{6}\right] \\
& \cdot\left(8 e^{y}+136 e^{2 y}+138 e^{3 y}+14 e^{4 y}\right) \\
& -\frac{\gamma^{2} t^{2}}{2}\left(1+\frac{\gamma t}{3}-\gamma\right)\left(4 e^{2 y}+6 e^{3 y}+2 e^{4 y}\right),
\end{aligned}
$$

and in the same way, we can find some more terms; therefore, we have

$$
\begin{aligned}
v(t, y)= & e^{y}-(1+\gamma t-\gamma)\left(2 e^{y}+e^{2 y}\right) \\
& +\left[(1-\gamma)^{2}+2 \gamma t(1-\gamma)+\frac{\gamma^{2} t^{2}}{2}\right] \\
& \cdot\left(4 e^{y}+14 e^{2 y}+3 e^{3 y}\right)-\left[(1-\gamma)^{3}\right. \\
& \left.+3 \gamma t\left(1-\gamma^{2}\right)+\frac{3}{2} \gamma^{2}(1-\gamma) t^{2}+\frac{\gamma^{3} t^{3}}{6}\right] \\
& \cdot\left(8 e^{y}+136 e^{2 y}+138 e^{3 y}+14 e^{4 y}\right) \\
& -\frac{\gamma^{2} t^{2}}{2}\left(1+\frac{\gamma t}{3}-\gamma\right)\left(4 e^{2 y}+6 e^{3 y}+2 e^{4 y}\right)+\cdots
\end{aligned}
$$




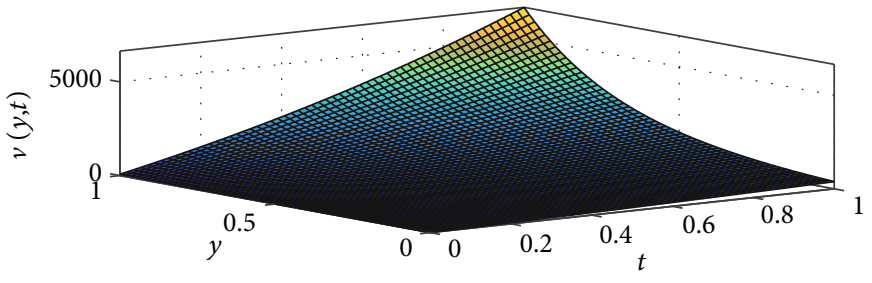

$(0.75)$

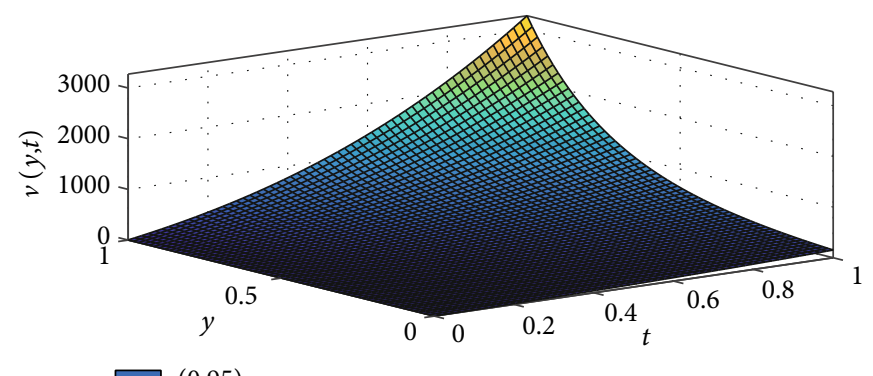

$(0.95)$

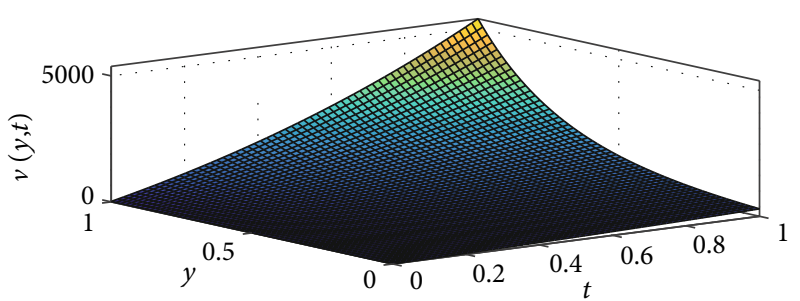

$(0.85)$

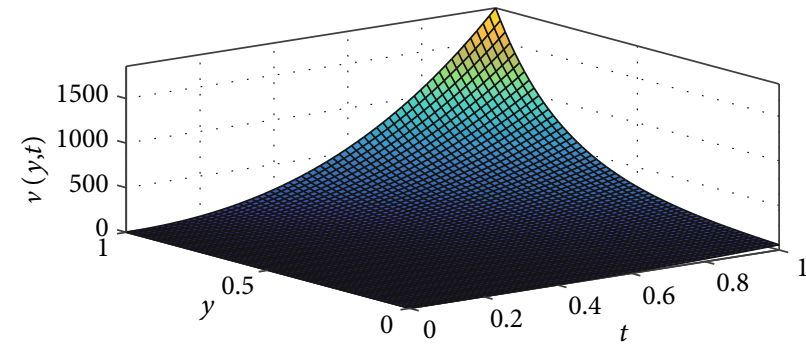

$(1.0)$

Figure 2: Surface plots of the resultant solution up to four terms at different values of $\gamma$ for Example 2.

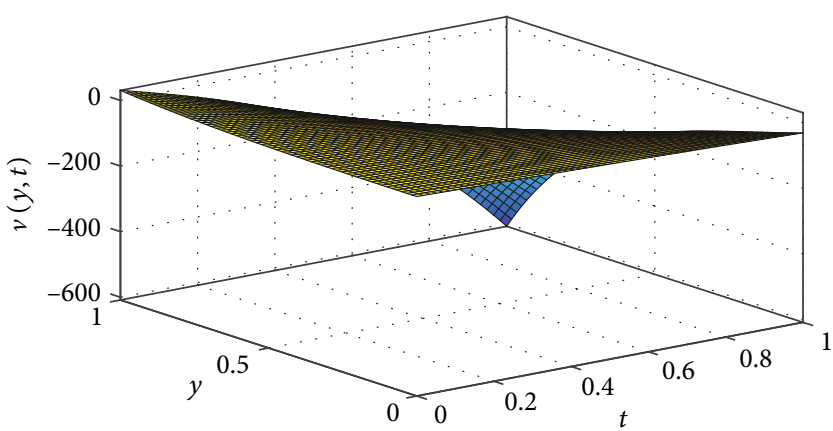

$(0.75)$

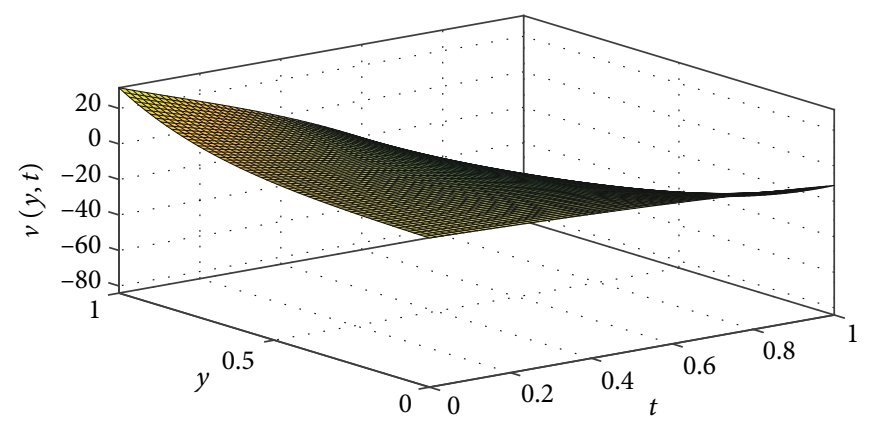

$(0.95)$

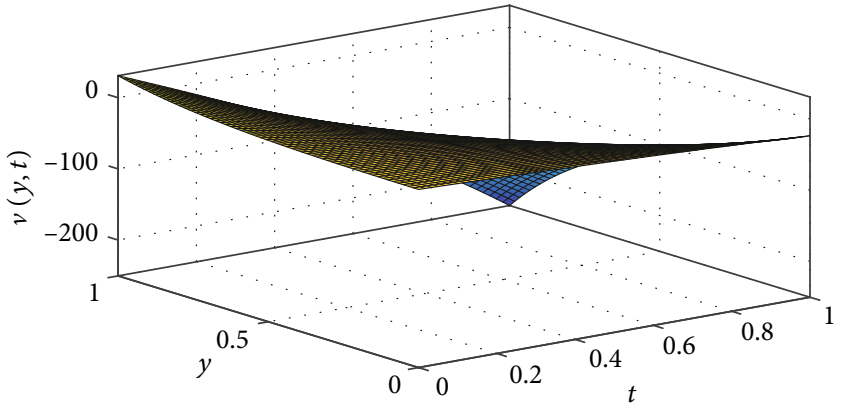

$(0.85)$

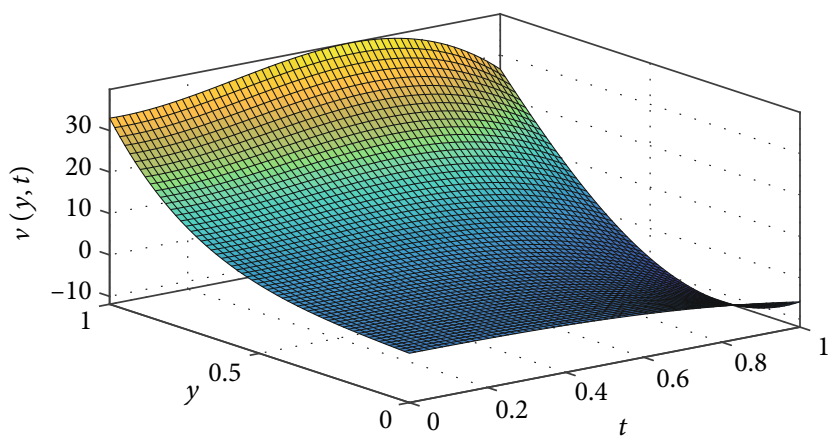

$(1.0)$

FIgURE 3: Surface plots of the resultant solution up to four terms at different values of $\gamma$ for Example 3.

Here, we plot the approximate solution of the FBBM equation up to four terms in Figure 2. The approximate solution graphs for various fractional orders are given in Figure 2. We see from graphs as the order $\gamma \longrightarrow 1$, the behavior of the surfaces of the solution tends to the integer order. If we put $\gamma=1$ in the approximate solution, we get the approximate solution at integer order.
Example 3. Consider the FBBM equation using CFFOD as

$$
\begin{aligned}
{ }^{C F} D_{t}^{\gamma} v(t, y)+v_{y}(t, y) & \\
+v(t, y) v_{y}(t, y)+v_{y y y}(t, y) & =0, \\
v(0, y) & =y^{2} .
\end{aligned}
$$


and in the same way, we can find some more terms; therefore, we have

$$
\begin{aligned}
v_{0}= & y^{2}, \\
v_{1}= & -(1+\gamma t-\gamma)\left(2 y+2 y^{3}\right), \\
v_{2}= & {\left[(1-\gamma)^{2}+2 \gamma t(1-\gamma)+\frac{\gamma^{2} t^{2}}{2}\right]\left(14^{2} y^{2}+4 y^{3}+6 y^{4}\right), } \\
v_{3}= & -\left[(1-\gamma)^{3}+3 \gamma t\left(1-\gamma^{2}\right)+\frac{3}{2} \gamma^{2}(1-\gamma) t^{2}+\frac{\gamma^{3} t^{3}}{6}\right] \\
& \cdot\left(24+200 y+12 y^{2}+88 y^{3}+20 y^{4}+48 y^{5}\right) \\
& -\frac{\gamma^{2} t^{2}}{2}\left(1+\frac{\gamma t}{3}-\gamma\right)\left(4 y+16 y^{3}+12 y^{5}\right) .
\end{aligned}
$$

Here, we plot the approximate solution of FBBM equation up to four terms in Figure 3. The approximate solution graphs for various fractional orders are given in Figure 3. We see from graphs as the order $\gamma \longrightarrow 1$, the behavior of the surfaces of the solution tends to the integer order solution. If we put $\gamma=1$ in the approximate solution, we get the approximate solution at the integer order for the same problem.

Example 4. Consider the modified FBBM equation using CFFOD as

$$
\begin{aligned}
{ }^{C F} D_{t}^{\gamma} v(t, y)+v_{y}(t, y) & \\
+v^{2}(t, y) v_{y}(t, y)+v_{y y y}(t, y) & =0, \\
v(0, y) & =e^{y} .
\end{aligned}
$$

With the help of the procedure mentioned in Case 3, we have

$$
\begin{aligned}
v_{0}= & e^{y}, \\
v_{1}= & -(1+\gamma t-\gamma)\left(2 e^{y}+e^{3 y}\right), \\
v_{2}= & {\left[(1-\gamma)^{2}+2 \gamma t(1-\gamma)+\frac{\gamma^{2} t^{2}}{2}\right]\left(4 e^{y}+36 e^{3 y}+5 e^{5 y}\right), } \\
v_{3}=- & {\left[(1-\gamma)^{3}+3 \gamma t\left(1-\gamma^{2}\right)+\frac{3}{2} \gamma^{2}(1-\gamma) t^{2}+\frac{\gamma^{3} t^{3}}{6}\right] } \\
& \cdot\left(8 e^{y}+1104 e^{3 y}+778 e^{5 y}+22 e^{7 y}\right) \\
& -\frac{\gamma^{2} t^{2}}{2}\left(1+\frac{\gamma t}{3}-\gamma\right)\left(12 e^{3 y}+20 e^{5 y}+7 e^{7 y}\right),
\end{aligned}
$$

and in the same way, we can find some more terms; therefore, we have

$$
\begin{aligned}
v(t, y)= & e^{y}-(1+\gamma t-\gamma)\left(2 e^{y}+e^{3 y}\right) \\
& +\left[(1-\gamma)^{2}+2 \gamma t(1-\gamma)+\frac{\gamma^{2} t^{2}}{2}\right] \\
& \cdot\left(4 e^{y}+36 e^{3 y}+5 e^{5 y}\right)--\left[(1-\gamma)^{3}\right. \\
& \left.+3 \gamma t\left(1-\gamma^{2}\right)+\frac{3}{2} \gamma^{2}(1-\gamma) t^{2}+\frac{\gamma^{3} t^{3}}{6}\right] \\
& \cdot\left(8 e^{y}+1104 e^{3 y}+778 e^{5 y}+22 e^{7 y}\right) \\
& -\frac{\gamma^{2} t^{2}}{2}\left(1+\frac{\gamma t}{3}-\gamma\right)\left(12 e^{3 y}+20 e^{5 y}+7 e^{7 y}\right)+\cdots
\end{aligned}
$$

Here, we plot the approximate solution of the FBBM equation up to four terms in Figure 4. The approximate solution graphs for various fractional orders are given in Figure 4. We see from graphs as the order $\gamma \longrightarrow 1$, the behavior of the surfaces of the solution tends to the integer order solution. Also, if we put $\gamma=1$ in the approximate solution, we get the approximate solution at integer order for the same problem.

Example 5. Consider the modified FBBM equation using CFFOD as

$$
\begin{aligned}
{ }^{C F} D_{t}^{\gamma} v(t, y)+v_{y}(t, y)+v^{2}(t, y) v_{y}(t, y)+v_{y y y}(t, y) & =0, \\
v(0, y) & =y^{2} .
\end{aligned}
$$

With the help of the procedure discussed for Case 3, one may have

$$
\begin{aligned}
v_{0}= & y^{2}, \\
v_{1}= & -(1+\gamma t-\gamma)\left(2 y+2 y^{5}\right), \\
v_{2}= & {\left[(1-\gamma)^{2}+2 \gamma t(1-\gamma)+\frac{\gamma^{2} t^{2}}{2}\right]\left(2+120 y^{2}+20 y^{4}+18 y^{8}\right), } \\
v_{3}= & -\left[(1-\gamma)^{3}+3 \gamma t\left(1-\gamma^{2}\right)+\frac{3}{2} \gamma^{2}(1-\gamma) t^{2}+\frac{\gamma^{3} t^{3}}{6}\right] \\
& \cdot\left(160+240 y+100 y^{3}+6786 y^{5}+344 y^{7}+244 y^{11}\right) \\
& -\frac{\gamma^{2} t^{2}}{2}\left(1+\frac{\gamma t}{3}-\gamma\right)\left(12 y^{3}+40 y^{7}+28 y^{11}\right),
\end{aligned}
$$

and in the same way, we can find the other terms. Therefore, we get

$$
\begin{aligned}
v(t, y)= & y^{2}-(1+\gamma t-\gamma)\left(2 y+2 y^{5}\right) \\
& \cdot\left[(1-\gamma)^{2}+2 \gamma t(1-\gamma)+\frac{\gamma^{2} t^{2}}{2}\right] \\
& \cdot\left(2+120 y^{2}+20 y^{4}+18 y^{8}\right) \\
& -\left[(1-\gamma)^{3}+3 \gamma t\left(1-\gamma^{2}\right)+\frac{3}{2} \gamma^{2}(1-\gamma) t^{2}+\frac{\gamma^{3} t^{3}}{6}\right] \\
& \cdot\left(160+240 y+100 y^{3}+6786 y^{5}+344 y^{7}+244 y^{11}\right) \\
& -\frac{\gamma^{2} t^{2}}{2}\left(1+\frac{\gamma t}{3}-\gamma\right)\left(12 y^{3}+40 y^{7}+28 y^{11}\right)+\cdots
\end{aligned}
$$




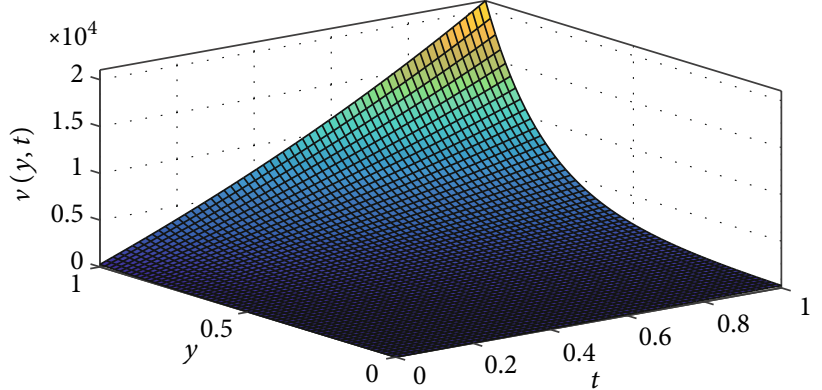

$(0.75)$

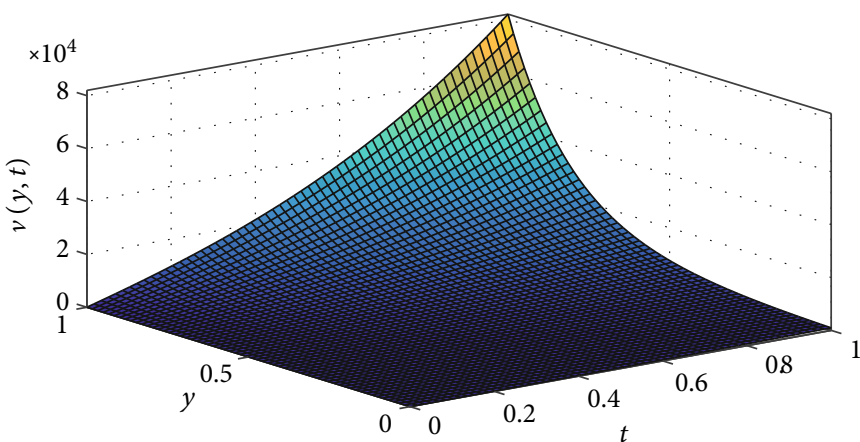

$(0.95)$

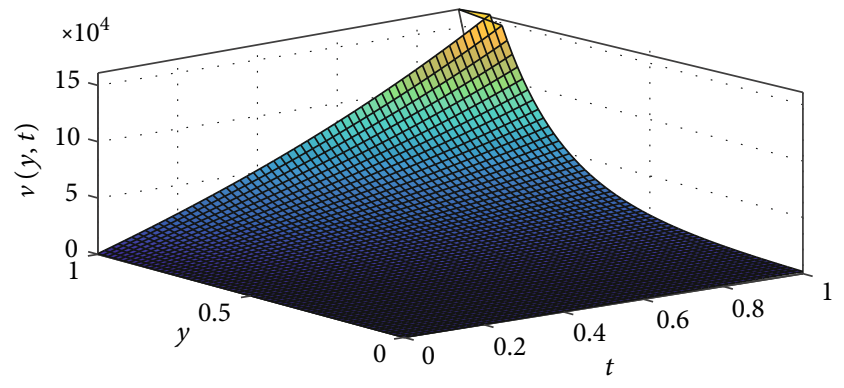

$(0.85)$

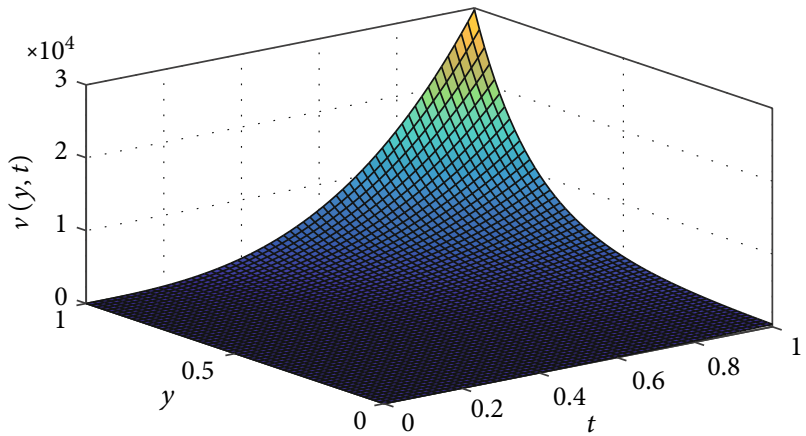

$(1.0)$

Figure 4: Surface plots of the resultant solution up to four terms at different values of $\gamma$ for Example 4 .
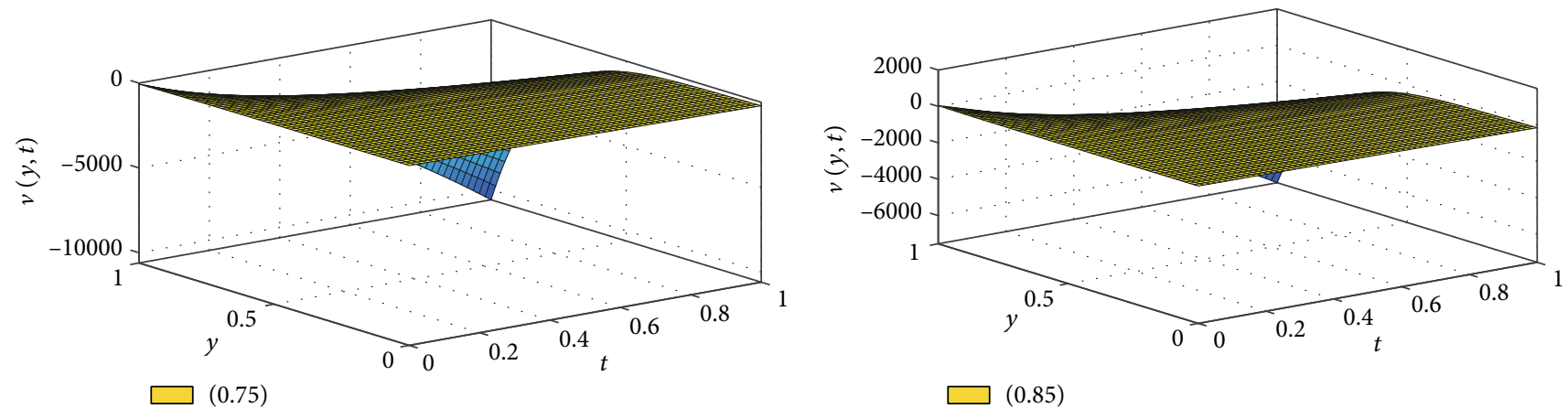

$(0.75)$

$(0.85)$

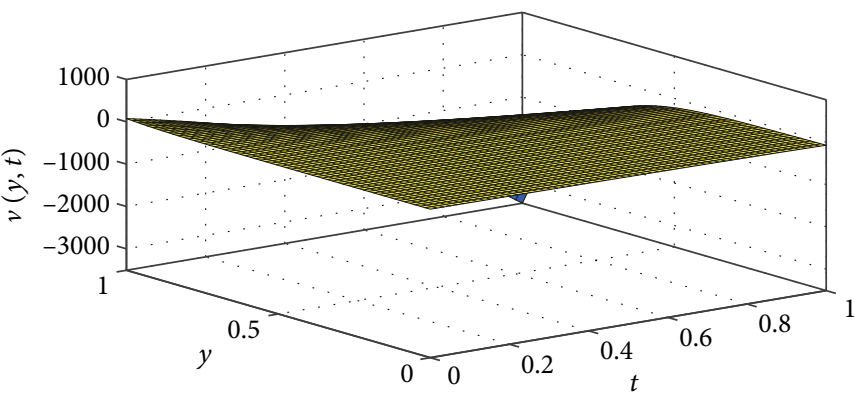

$(0.95)$

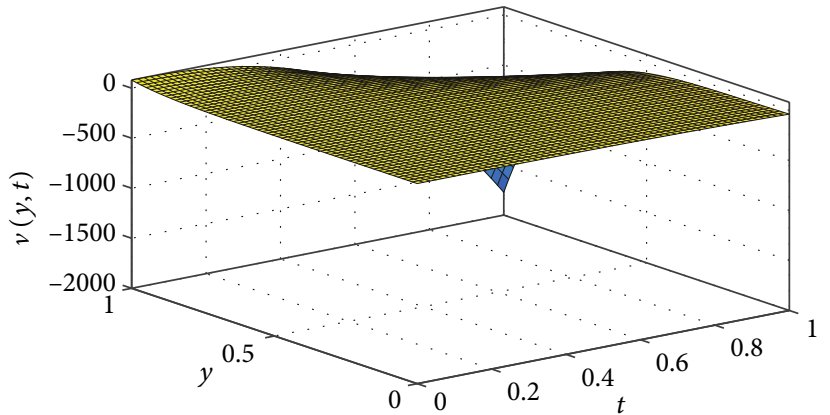

$(1.0)$

Figure 5: Surface plots of the resultant solution up to four terms at different values of $\gamma$ for Example 5.

Here, we plot the approximate solution of FBBM equation up to four terms in Figure 5. The approximate solution graphs for various fractional orders are given in Figure 5. We see from graphs as the order $\gamma \longrightarrow 1$, the behavior of the surfaces of the solution tends to the integer order solution. Also, if we put $\gamma=1$ in the approximate solution, we get the approximate solution at integer order for the same problem. 


\section{Conclusion}

In our work, some existence results about the solution to the nonlinear problem of BBM equations under nonsingular kernel-type derivative have been developed successfully. We have discussed different cases of the concerned equations for semianalytical results. For approximate analytical results, a novel iterative method of Laplace transform coupled with Adomian polynomials has been used. Further, by providing an example, we have computed the absolute errors in comparison with VHPM for first four-term solutions at different values of variables $t$ and $y$ against $\gamma=1$. We observed that the absolute error is slightly good than the mentioned VHPM. Therefore, the concerned method of LADM can be used as a powerful tool to handle many nonlinear problems of FODEs. Since, the aforementioned equations are increasingly used to model numerous phenomena of physics including the propagation of heat or sound waves, fluid flow, elasticity, electrostatics, and electrodynamics, and population dynamics in biology. A large numbers of the aforementioned equations may be used in fluid mechanics and hydrodynamics. Since fractional derivatives have a greater degree of freedom and produce the complete spectrum of the physical phenomenon which include the ordinary derivative as particular case, global dynamics of the aforesaid physical phenomenon may be investigated. Since the BBM equation can also be used to model various physical systems like acoustic-gravity waves in compressible fluids, acoustic waves in enharmonic crystals, the hydromagnetic waves in cold plasma, (see [41]), investigation of the BBM equation and its various cases under different fractional order derivatives may be lead us to investigate some more comprehensive results by using various fractional orders which will include the classical order solution as a special case. The nonlocal behaviors of such problems can be well studied by using nonsingular fractional order derivative. In the future, the concerned BBM equation can be investigated by using more general fractional order derivative with nonsingular kernel of the Mittag-Leffler function.

\section{Data Availability}

Data availability is not applicable in this manuscript.

\section{Conflicts of Interest}

There is no competing interest regarding this work.

\section{Authors' Contributions}

An equal contribution has been done by all the authors.

\section{Acknowledgments}

Prince Sultan University provided support through the research group Nonlinear Analysis Methods in Applied Mathematics (NAMAM), group number RG-DES-201701-17.

\section{References}

[1] A. A. Kilbas, O. I. Marichev, and S. G. Samko, Fractional Integrals and Derivatives Theory and Applications, Gordon and Breach, Switzerland, 1993.

[2] K. S. Miller and B. Ross, An Introduction to the Fractional Calculus and Fractional Differential Equations, Wiley, New York, NY, USA, 1993.

[3] I. Podlubny, Fractional Differential Equations, Mathematics in Science and Engineering, Academic Press, New York, NY, USA, 1999.

[4] A. A. Kilbas, H. M. Srivastava, and J. J. Trujillo, Theory and Applications of Fractional Differential Equations, Elsevier, Amsterdam, 2006.

[5] M. Benchohra, J. R. Graef, and S. Hamani, "Existence results for boundary value problems with nonlinear fractional differential equations," Applicable Analysis, vol. 87, pp. 851-863, 2008.

[6] A. Shaikh, A. Tassaddiq, K. S. Nisar, and D. Baleanu, "Analysis of differential equations involving Caputo-Fabrizio fractional operator and its applications to reaction-diffusion equations," Advances in Difference Equations, vol. 2019, no. 1, 14 pages, 2019.

[7] Y. S. Özkan, E. Yaşar, and A. R. Seadawy, "A third-order nonlinear Schrödinger equation: the exact solutions, groupinvariant solutions and conservation laws," Journal of Taibah University for Science, vol. 14, no. 1, pp. 587-597, 2020.

[8] H. Ahmad, A. R. Seadawy, T. A. Khan, and P. Thounthong, "Analytic approximate solutions for some nonlinear Parabolic dynamical wave equations," Journal of Taibah University for Science, vol. 14, no. 1, pp. 346-358, 2020.

[9] E. S. Selima, A. R. Seadawy, and X. Yao, "The nonlinear dispersive Davey-Stewartson system for surface waves propagation in shallow water and its stability," The European Physical Journal Plus, vol. 131, no. 12, pp. 1-16, 2016.

[10] A. H. Khater, D. K. Callebaut, and A. R. Seadawy, "General soliton solutions for nonlinear dispersive waves in convective type instabilities," Physica Scripta, vol. 74, no. 3, pp. 384-393, 2006.

[11] A. R. Seadawy, "Three-dimensional weakly nonlinear shallow water waves regime and its traveling wave solutions," International Journal of Computational Methods, vol. 15, no. 03, article 1850017, 2018.

[12] K. Shah, M. A. Alqudah, F. Jarad, and T. Abdeljawad, "Semianalytical study of Pine Wilt Disease model with convex rate under Caputo-Febrizio fractional order derivative," Chaos, Solitons \& Fractals, vol. 135, article 109754, 2020.

[13] K. Shah, T. Abdeljawad, I. Mahariq, and F. Jarad, "Qualitative analysis of a mathematical model in the time of COVID-19," BioMed Research International, vol. 2020, Article ID 5098598, 11 pages, 2020.

[14] A. R. Seadawy and S. Z. Alamri, "Mathematical methods via the nonlinear two-dimensional water waves of Olver dynamical equation and its exact solitary wave solutions," Results in Physics, vol. 8, pp. 286-291, 2018.

[15] A. Ali, A. R. Seadawy, and D. Lu, "New solitary wave solutions of some nonlinear models and their applications," Advances in Difference Equations, vol. 2018, no. 1, 2018.

[16] M. Arshad, D. Lu, J. Wang, and Abdullah, "Exact traveling wave solutions of a fractional Sawada-Kotera equation," East Asian Journal on Applied Mathematics, vol. 8, no. 2, pp. 211223, 2019. 
[17] T. Abdeljawad and D. Baleanu, "On fractional derivatives with exponential kernel and their discrete versions," Reports on Mathematical Physics, vol. 80, no. 1, pp. 11-27, 2017.

[18] T. Abdeljawad and D. Baleanu, "Discrete fractional differences with nonsingular discrete Mittag-Leffler kernels," Advances in Difference Equations, vol. 2016, no. 1, 2016.

[19] M. Al-Refai and T. Abdeljawad, "Analysis of the fractional diffusion equations with fractional derivative of non-singular kernel," Advances in Difference Equations, vol. 2017, no. 1, 2017.

[20] F. Jarad and T. Abdeljawad, "Generalized fractional derivatives and Laplace transform," Discrete \& Continuous Dynamical Systems S, vol. 13, no. 3, pp. 709-722, 2020.

[21] A. H. Arnous, A. R. Seadawy, R. T. Alqahtani, and A. Biswas, "Optical solitons with complex Ginzburg-Landau equation by modified simple equation method," Optik, vol. 144, pp. 475-480, 2017.

[22] D. Lu, C. Yue, and M. Arshad, "Traveling wave solutions of space-time fractional generalized fifth-order KdV equation," Advances in Mathematical Physics, vol. 2017, Article ID 6743276, 6 pages, 2017.

[23] V. Lakshmikantham, S. Leela, and J. Vasundhara, Theory of fractional dynamic systems, Cambridge Academic Publishers, Cambridge, UK, 2009.

[24] R. Hilfer, Applications of Fractional Calculus in Physics, World Scientific, Singapore, 2000.

[25] T. B. Benjamin, J. L. Bona, and J. J. Mahony, "Model equations for long waves in nonlinear dispersive systems," Philosophical Transactions of the Royal Society A: Mathematical and Physical Sciences, vol. 272, no. 1220, pp. 47-78, 1997.

[26] M. Molati and C. M. Khalique, "Lie symmetry analysis of the time-variable coefficient B-BBM equation," Advances in Difference Equations, vol. 2012, no. 1, 2012.

[27] K. Singh and R. K. Gupta, "Lie symmetries and exact solutions of a new generalized Hirota-Satsuma coupled KdV system with variable coefficients," International Journal of Engineering Science, vol. 44, no. 3-4, pp. 241-255, 2006.

[28] A. Abdullah, R. Seadawy, and W. Jun, "Mathematical methods and solitary wave solutions of three-dimensional ZakharovKuznetsov-Burgers equation in dusty plasma and its applications," Results in Physics, vol. 7, pp. 4269-4277, 2017.

[29] D. Lu, A. R. Seadawy, and A. Ali, "Applications of exact traveling wave solutions of modified Liouville and the symmetric regularized long wave equations via two new techniques," Results in Physics, vol. 9, pp. 1403-1410, 2018.

[30] D. Lu, A. R. Seadawy, and A. Ali, "Dispersive traveling wave solutions of the Equal-Width and Modified Equal-Width equations via mathematical methods and its applications," Results in Physics, vol. 9, pp. 313-320, 2018.

[31] D. Lu, A. R. Seadawy, and A. Ali, "Structure of traveling wave solutions for some nonlinear models via modified mathematical method," Open Physics, vol. 16, no. 1, pp. 854-860, 2018.

[32] A. R. Seadawy and K. El-Rashidy, "Dispersive solitary wave solutions of Kadomtsev-Petviashvili and modified KadomtsevPetviashvili dynamical equations in unmagnetized dust plasma," Results in Physics, vol. 8, pp. 1216-1222, 2018.

[33] M. Wang and Y. Wang, "A new Backlund transformation and multi-soliton solutions to the KdV equation with general variable coefficients," Physics Letters A, vol. 287, no. 3-4, pp. 211216, 2001.
[34] M. Arshad, D. Lu, and J. Wang, " $(N+1)$-dimensional fractional reduced differential transform method for fractional order partial differential equations," Communications in Nonlinear Science and Numerical Simulation, vol. 48, pp. 509-519, 2017.

[35] K. Singh, R. K. Gupta, and S. Kumar, "Benjamin-BonaMahony (BBM) equation with variable coefficients: similarity reductions and Painlevé analysis," Applied Mathematics and Computation, vol. 217, no. 16, pp. 7021-7027, 2011.

[36] A. M. Wazwaz, "New travelling wave solutions of different physical structures to generalized BBM equation," Physics Letters $A$, vol. 355, no. 4-5, pp. 358-362, 2006.

[37] M. Caputo and M. Fabrizio, "A new definition of fractional derivative without singular kernel," Progress in Fractional Differentiation and Applications, vol. 1, pp. 73-85, 2015.

[38] T. Bashiri, S. M. Vaezpour, and J. J. Nieto, “Approximating solution of Fabrizio-Caputo Volterra's model for population growth in a closed system by homotopy analysis method," Journal of Function Spaces, vol. 2018, Article ID 3152502, 10 pages, 2018.

[39] T. A. Burton, "A fixed-point theorem of Krasnoselskii," Applied Mathematics Letters, vol. 11, no. 1, pp. 85-88, 1998.

[40] F. H. Easif, S. A. Manaa, B. A. Mahmood, and M. A. Yousif, "Variational homotopy perturbation method for solving Benjamin-Bona-Mahony equation," Applied Mathematics, vol. 6, no. 4, pp. 675-683, 2015.

[41] D. Daghan, H. Yavuz Mart, and G. Yildiz, "Applications of homotopy perturbation method for nonlinear partial differential equations," WSEAS Transactions on Mathematics, vol. 16, pp. 276-282, 2017. 